\title{
AHP-Based Resource Utilization Scheme at the Network Edge with Ad Hoc Network Gateway
}

\author{
Waesomesudin Waedorkor and Suntorn Witosurapot
}

\begin{abstract}
A sensitive problem in active learning classroom affecting the performance of Internet video streams is caused from the network degradation during the times of overloaded traffics. In this paper, we investigate how such a problem can be solved by a mean of edge computing system running at a gateway device so that virtualized services for transcoded video streams can be offered at the local ad-hoc network. In addition, we explore the use of multi-criteria decision making mechanism with AHP method for suggesting sensible network resources that can yield possible video qualities. Based on the result obtained by empirical network traces on a video clip transfer, our proposed solution selects lower levels of network utilizations than those of DASH server for similar video resolutions and frame-rates. Hence, it is beneficial for empowering the teacher to deploy flexible network policies in times of congestion at ease so that the residue of network resource can be given to some other essential tasks.
\end{abstract}

Index Terms-Active learning, adaptive video streaming, AHP, edge computing, DASH service.

\section{INTRODUCTION}

Active learning strategies often demand large volumes of video streaming to conduct the learner-centric style of education. In this regard, the network quality becomes a prime concern since it will play a great impact to learner's Quality of Experience (QoE) [1]. If the network connection becomes overloaded during a surge of concurrent learners, unpleasant visualizations can be experienced on the display device, such as delayed frames or frame skips, etc. For a clear illustration, let see Fig. 1, which illustrates a case of 5 similar learners working in a local network and competing for bandwidth on the access of video contents from external web sites. As seen in the graph of Fig. 2(a), when the network is under-utilized (during the period of $0-80$ second), the network bandwidth given to each learner is shared adequately (but at different levels depending on the number of new learner coming in every 10 second). As consequence, the delay times for moving pictures are fluctuated, but remains in an acceptable range so no annoyance to users, as seen in Fig. 2(b). However, during the time at 100 second onward, the network status turns to an overloaded condition. So, all learners will be in jeopardy since an adequate amount of bandwidth, as well as acceptable delay, will be no longer received for smoothing the video views.

Manuscript received February 12, 2018; revised March 28, 2018.

Waesomesudin Waedorkor and Suntorn Witosurapot are with the Department of Computer Engineering, Prince of Songkla University, Hatyai, Songkhla, Thailand (e-mail: waesomesudin.w@yru.ac.th, wsuntorn@coe.psu.ac.th).

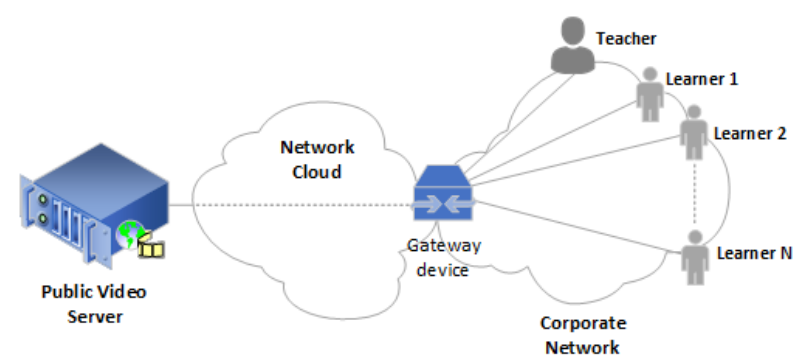

Fig. 1. Motivated network scenario.

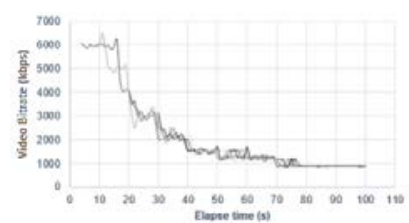

(a) Bandwidth

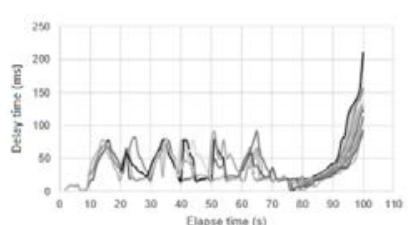

(b) Transmission delay
Fig. 2. Network performance metrics.

By investigating the above example, a direct solution can be obtained by a) reducing the amount of network traffic occurring on the external link, and b) allowing a sort of proxy mechanism to be devised for local video access. The contribution of this paper is then on a promising solution that attempts to minimize the incurred traffic of video accesses from the public web sites by exploiting a) Edge computing and b) Docker with proxy-alike capability. Nevertheless, some form of decision making mechanism will be needed in order to release the burden of finding suitable Docker's configurations in response to the dynamics in the network communication channel.

The structure of this paper is as follows: the Section II will provide a background of video adaptation technique, its compatible server, Edge computing and AHP method, which motivates our proposed solution. Then, the Section III presents the proposed architecture, as well as its internal processes, following with experiments in the Section IV. Finally, the Section V concludes the paper.

\section{BACKGROUND}

\section{A. Video Adaptation and DASH Server}

Internet video contents can adapt both their resolutions and frame rates in response to the network fluctuation. To realize this adaptability feature at a video server, the technique of Dynamic Adaptive Streaming over HTTP (DASH) [1], [2] will be often required. In essence, a file of video and audio contents will be divided into a set of segments, where each segment is regenerated into various frame-rates, resolutions and therefore, different playback length. As shown in Table I, they are typical factors of video/audio characteristics that are 
available in the DASH server of YouTube service [3]. Depending on the available bandwidth, these factors will be considered on the DASH client's device so that appropriate bit-rate segments can be selected on the DASH server and delivered to the client. By using the mean of the adaptive bit-rate delivery on all video segments, it can be assured that high user-perceived QoE [4], [5] will be available, but on the expense of compromised video qualities under given limited network resources.

TABLE I: PARTIAL STANDARD VIDEO/AUDIO CHARACTERISTICS RECOMMENDED BY YOUTUBE [3]

\begin{tabular}{cccc}
\hline \multicolumn{4}{c}{ Rideo } \\
$\begin{array}{c}\text { RECOMMENDED BY YOUTUBE [3] } \\
\text { (Fps) }\end{array}$ & $\begin{array}{c}\text { Video } \\
\text { resolution } \\
\text { (Pixel) }\end{array}$ & $\begin{array}{c}\text { Audio } \\
\text { bit-rate } \\
\text { (Kbps) }\end{array}$ & $\begin{array}{c}\text { Video bit-rate } \\
\text { (Mbps) }\end{array}$ \\
\hline 30 & 480 & 32 & 2.5 \\
60 & 480 & 128 & 4 \\
30 & 720 & 32 & 5 \\
60 & 720 & 128 & 7.5 \\
30 & 1080 & 32 & 10 \\
60 & 1080 & 128 & 15 \\
\hline \hline
\end{tabular}

\section{B. Edge Computing Approach}

Owing to the fact of adaptive bit-rate delivery mentioned above, segments of the divided video contents are independent from each other and their characteristics may not necessarily be the same when spreading over the set of servers in the Internet. This style of operations has motivated the Edge Computing (EC) concept [4], [6] for enabling the computing facility at the edge of Internet (i.e. near or at the corporate network), rather than locating somewhere in the Internet as found in typical Cloud Computing. In this regard, it will promote the alternative use of local services for a given corporate, hence produces little transmission latency for their users and low bandwidth consumption on the Internet access of the corporate link. In this work, the EC is used to push the adaptive video delivery service away from a DASH server to the network edge (i.e. at the device locating at the border of a corporate network), therefore deteriorating the external access of required video contents from corporate users. In this regard, the Docker technology is well-suited [7], [8] since it can enable the replications of some of cloud services [7] or video transcoding micro-service platform [8], [9] at ease and in a quick manner.

\section{AHP Method}

Regarding the factors of video/audio characteristics of video segments found in Table I, different combinations of them will provide a variety of different services. Since the DASH server is likely to select the combination that will be best-fitted to the current bandwidth availability, this may not be the case where tolerant bandwidth utilization is preferred. For instance, in the context of embedded server, where precious network resource may be needed for serving some other concurrent tasks, besides the adaptive video service. In this work, we consider how to get the better choices of combined factors in the preferred case. By viewing this consideration as a basic problem of multi-criteria decision making (MCDM) system, it looks promising that an optimal set of required solutions can be resulted. Our focus is on the Analytical Hierarchical Process (AHP) method [10], [11] since it is convenient to use and allows both qualitative and quantitative factors to be computed nicely together.

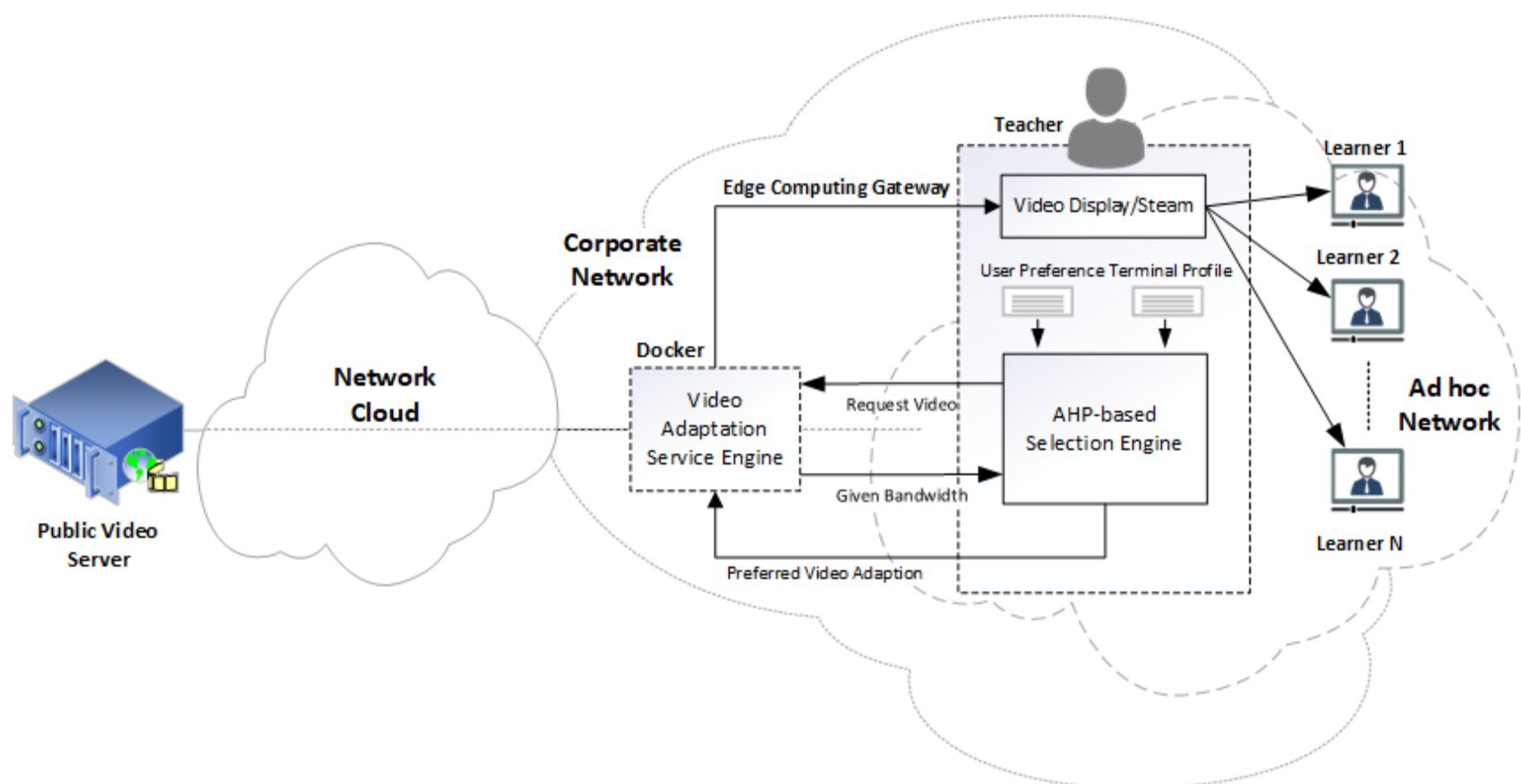

Fig. 3. Proposed architecture of smart gateway.

\section{PROPOSED ARCHITECTURE}

In this section, we describe our conceptual architecture of a smart gateway with edge service provision, where its functionality is mainly handled by a) Video Adaptation Service Engine for performing EC services to transcode videos from a remote DASH server, and b) AHP-based Adaptation Selection Engine for analyzing the characteristics of video contents that are well-matched for the current network condition with respect to user requirements. From the technical viewpoint, the co-operations of these two 
engines are rather straightforward and follows closely to the transcoding workflow given in [8] for replicating adaptive video delivery service from a DASH server to the corporate network. However, in the context of this paper, we will give the highlight on the AHP-based Adaptation Selection Engine for suggesting the lower network resource utilization than those required by typical DASH server.

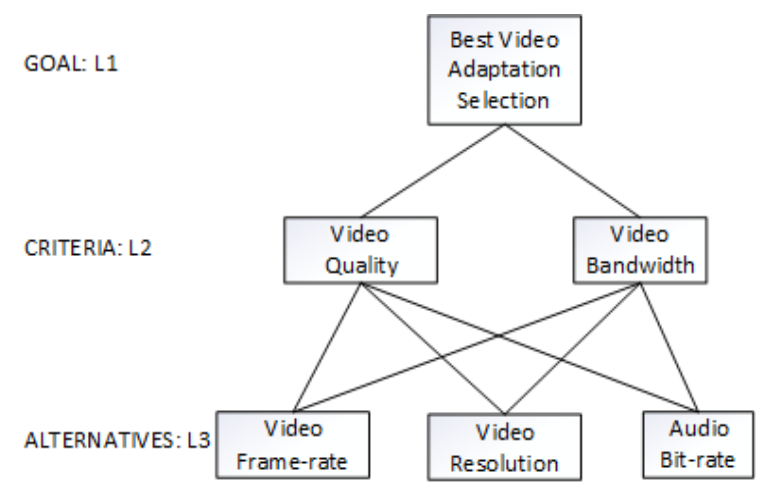

Fig. 4. Decision hierarchy.

In our proposed architecture, the adaptation selection engine will decide on what level the network resource should be optimally consumed, regarding the network utilization policy. Here, two policies are considered; maximizing video qualities, and minimizing video bandwidth consumption. In this regard, the performance metrics of video frame-rate, video resolution, and audio bit-rate will be considered as alternatives in Level 3 of a hierarchical model (see Fig. 4). While Level 2 contains all the criteria (i.e. two desired policies), the topmost of Level 1 specifies the ultimate goal of finding the best video adaptive selection. Regarding the AHP process [11] in Fig. 5, the matric of criteria in both cases can be formed as shown in Table II. Here, we assume that the Video Quality in the case I is valued 5 times of Video Bandwidth (out of 10 scale) and vice versa in the case II. In Table III, the matric of alternatives assumes that the Video frame-rate in the case I is valued 7 times of Video Resolution and 5 times of Audio bit-rate. In contrast, in the case II, the value of Video frame-rate is assumed to be $1 / 3$ times of Video Resolution and 2 times of Audio bit-rate. After finishing the AHP calculation (see detailed steps in [11]), the final weights of all alternatives, as well as their ranking, will be obtained as shown in Table IV, and calculated further to find out the Performance Indexes of possible combinations of video performance matric as shown in Table V. Noticed that the better value is recommend to those of which value is closer to 1 [11].

\section{PERFORMANCE EVAluATION}

To evaluate the performance of the proposed smart gateway, we conduct an experiment in a simulated corporate environment of a school network, where the teacher of an ad hoc network aims to provide a better service of video accesses for their active learning students. In this experiment, the adaptive video streaming of Big Buck Bunny [3] on the YouTube web site has been using throughout the experiments.

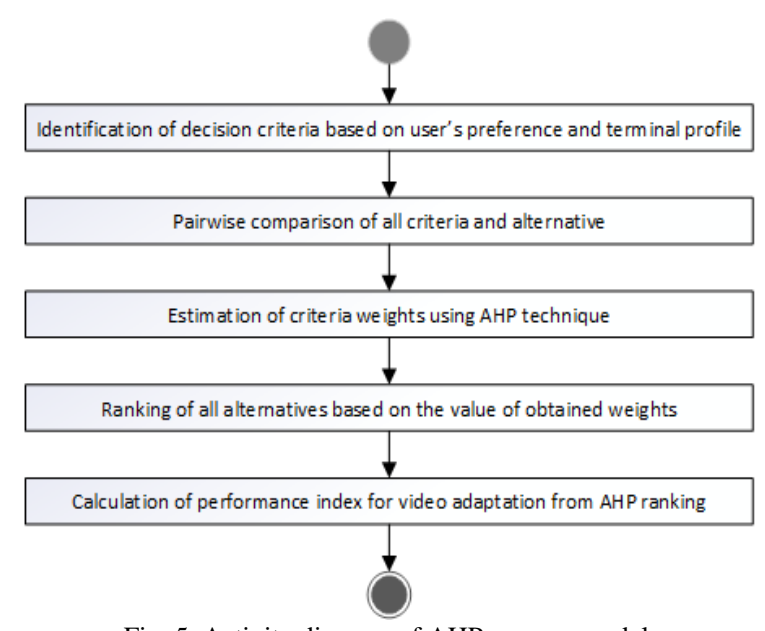

Fig. 5. Activity diagram of AHP process model.

TABLE II: MATRICES FOR CRITERIA

\begin{tabular}{ccccccc}
\hline \hline $\begin{array}{c}\text { Criteria } \\
\text { (Case I) }\end{array}$ & VQ & VB & & $\begin{array}{c}\text { Criteria } \\
\text { (Case II) }\end{array}$ & VQ & VB \\
\cline { 1 - 2 } \cline { 5 - 6 } VQ & 1 & 5 & & VQ & 1 & $1 / 5$ \\
VB & $1 / 5$ & 1 & VB & 5 & 1 \\
\hline \hline
\end{tabular}

Abbreviation: VQ: Video Quality, VB: Video Bandwidth

TABLE III: MATRICES FOR ALTERNATIVES

\begin{tabular}{ccccccccc}
\hline \hline Case I & VF & VR & AB & & Case II & VF & VR & AB \\
\cline { 1 - 4 } \cline { 5 - 8 } VF & 1 & 7 & 5 & & VF & 1 & $1 / 3$ & 2 \\
VR & $1 / 7$ & 1 & 5 & & VR & 3 & 1 & 3 \\
$\mathrm{AB}$ & $1 / 5$ & $1 / 5$ & 1 & & $\mathrm{AB}$ & $1 / 2$ & $1 / 3$ & 1 \\
\hline \hline
\end{tabular}

Abbreviation: VF: Video Frame-rate, VR: Video Resolution and AB: Audio Bit-rate

TABLE IV: RESULTED WEIGHTS \& RANKING

\begin{tabular}{ccccc}
\multicolumn{5}{c}{ TABLE IV: RESULTED WEIGHTS \& RANKING } \\
\hline \hline $\begin{array}{c}\text { Alternative } \\
\text { Result }\end{array}$ & $\begin{array}{c}\text { Weights } \\
\text { Case I }\end{array}$ & $\begin{array}{c}\text { Weights } \\
\text { Case II }\end{array}$ & $\begin{array}{c}\text { Ranking } \\
\text { Case I }\end{array}$ & $\begin{array}{c}\text { Ranking } \\
\text { Case II }\end{array}$ \\
\hline VF & 0.591 & 0.320 & 1 & 2 \\
VR & 0.316 & 0.534 & 2 & 1 \\
AB & 0.093 & 0.146 & 3 & 3 \\
\hline \hline
\end{tabular}

TABLE V: PERFORMANCE INDEX TEST OF CASE I \& II

\begin{tabular}{cccccc}
\hline \hline $\begin{array}{c}\text { Test Case } \\
\text { (Mbps) }\end{array}$ & $\begin{array}{c}\text { Adaptation Factors } \\
\text { Video } \\
\text { frame-rate }\end{array}$ & $\begin{array}{c}\text { Video } \\
\text { resolution }\end{array}$ & $\begin{array}{c}\text { Audio } \\
\text { bit-rate }\end{array}$ & $\begin{array}{c}\text { Performance } \\
\text { Index } \\
\text { (Case I) }\end{array}$ & $\begin{array}{c}\text { Performance } \\
\text { Index } \\
\text { (Case II) }\end{array}$ \\
\hline \multirow{3}{*}{3.5} & 60 & 480 & 32 & 0.997 & 0.901 \\
& 30 & 720 & 32 & 1.996 & 1.000 \\
& 60 & 720 & 128 & 2.011 & 1.996 \\
\hline \multirow{2}{*}{7} & 60 & 720 & 32 & 1.000 & 0.505 \\
& 30 & 1080 & 32 & 1.123 & 0.998 \\
& 60 & 1080 & 128 & 2.226 & 1.123 \\
\hline \multirow{2}{*}{13} & 60 & 1080 & 32 & 0.893 & 0.726 \\
& 30 & 1440 & 32 & 1.999 & 1.000 \\
& 60 & 1440 & 128 & 2.352 & 1.999 \\
\hline \hline
\end{tabular}

Based on the results obtained from our experiments as shown in Table VI, it can be noticed that indirect-accessing YouTube video via our proposed AHP-based gateway on both policies can yield lower level of utilized bandwidth than the direct-access counterpart. In addition, depending on the applied policy, the effective bandwidth utilization will be conformed as expected. For example, at the video matric of 720p, the bandwidth of 3.488 Mbps will be selected for streaming video at $30 \mathrm{fps}$ in the VB policy, but significantly changed to $6.144 \mathrm{Mbps}$ for streaming video at $60 \mathrm{fps}$ in the VQ policy. However, compared with the direct access from YouTube web site, they would consume higher amount of bandwidth in both cases (1.356 Mbps and 1.512 Mbps in the VQ and VB policy respectively. 
TABLE VI: COMPARISON OF DIFFERENT VIDEO/AUDIO BIT-RATES IN EXPERIMENTS

\begin{tabular}{|c|c|c|c|c|c|c|}
\hline $\begin{array}{c}\text { Video } \\
\text { Frame-rate } \\
\text { (Fps) }\end{array}$ & $\begin{array}{l}\text { Video } \\
\text { Resolution } \\
\text { (Pixel) }\end{array}$ & $\begin{array}{l}\text { Audio } \\
\text { Bit-rate } \\
\text { (Kbps) }\end{array}$ & $\begin{array}{l}\text { Direct Access } \\
\text { from YouTube } \\
\text { (Mbps) }\end{array}$ & $\begin{array}{c}\text { Indirect access via } \\
\text { AHP-based gateway } \\
\text { (Case I: VQ policy) } \\
\text { (Mbps) }\end{array}$ & $\begin{array}{c}\text { Indirect access via } \\
\text { AHP-based gateway } \\
\text { (Case II: VB policy) } \\
\text { (Mbps) }\end{array}$ & $\begin{array}{c}\text { Lower bandwidth } \\
\text { utilization } \\
\text { (Mbps) }\end{array}$ \\
\hline 60 & 480 & 128 & 4 & 3.106 & - & 0.894 \\
\hline 30 & 720 & 32 & 5 & - & 3.488 & 1.512 \\
\hline 60 & 720 & 128 & 7.5 & 6.144 & - & 1.356 \\
\hline 30 & 1080 & 32 & 8 & - & 7.108 & 0.892 \\
\hline 60 & 1080 & 128 & 12 & 10.68 & - & 1.32 \\
\hline 30 & 1440 & 32 & 16 & - & 13.856 & 2.144 \\
\hline
\end{tabular}

\section{CONCLUSION}

This paper has described how to decrease the congestion level occurred in a link at the Internet gateway of a corporate network when a large number of video demands are risen from internal users. By taking the mutual advantages of Edge computing via Docker replication service and AHP-based Adaptation Selection Engine, the utilized bandwidth on the external link can be effectively controlled according to the governed policy on local video transcoding service. For the future work, we plan to investigate the performance of our proposed gateway architecture in the IoT environment, where the bandwidth resource seems to be precious for accommodating a number of concurrent tasks in an expectable manner.

\section{REFERENCES}

[1] S. Zhao, Z. Li, D. Medhi, P. Lai, and S. Liu, "Study of user QoE improvement for dynamic adaptive streaming over HTTP (MPEG-DASH)," in Proc. Comp., Networking and Comm. (ICNC), Int. Conf., 2017, pp. 566-570.

[2] D. O. Trujillo, G. E. C. Golondrino, D. F. D. Dorado, W. Y. C. Muñoz, and J. L. A. Herrera, "Coding multimedia content using DASH standard,” pp. 1-7, 2016.

[3] Recommended upload encoding settings - YouTube Help. [Online]. Available: https://support.google.com/youtube/answer/1722171?hl=en

[4] Y. Li, P. A. Frangoudis, Y. Hadjadj-Aoul, and P. Bertin, "A mobile edge computing-based architecture for improved adaptive HTTP video delivery," in Proc. Int. Conf. Standards for Comm. and Networking, 2016, pp. 1-6.

[5] L. Van Ma, J. Park, J. Nam, J. Jang, and J. Kim, "An efficient scheduling multimedia transcoding method for DASH streaming in cloud environment,” Cluster Computing., 2017, pp. 1-11.

[6] T. Taleb, K. Samdanis, B. Mada, H. Flinck, S. Dutta, and D. Sabella, "On multi-access edge computing: A survey of the emerging $5 \mathrm{G}$ network edge architecture \& orchestration,” IEEE Comm. Surveys Tutorials., p. 1, 2017.

[7] B. I. Ismail et al., "Evaluation of docker as edge computing platform," in Proc. 2015 IEEE Conf. on Open Systems, 2015, pp. 130-135.
[8] O. Barais, J. Bourcier, Y.-D. Bromberg, and C. Dion, "Towards microservices architecture to transcode videos in the large at low costs," in Proc. 2016 Int. Conf. on Telecom. and Multimedia (TEMU), 2016, pp. 1-6.

[9] X. Wang, C. Wang, X. Zhou, and Z. Yang, "Video coding based on shape-adaptive all phase biorthogonal Transform and MPEG-4," J. of Communications, 2015, vol. 10, pp. 1004-1011.

[10] A. Rueangprathum, S. Limsiroratana, and S. Witosurapot, "User-driven multimedia adaptation framework for context-aware learning content service,” J. of Advances in Info. Tech., 2016, vol. 7, no. 3, pp. 182-185.

[11] A. Rueangprathum, S. Limsiroratana, and S. Witosurapot, "AHP-based adaptive resource selection for cognitive platform in cloud gaming service,” GSTF J. on Computing (JoC), vol. 4, no. 4, p. 52, 2016.

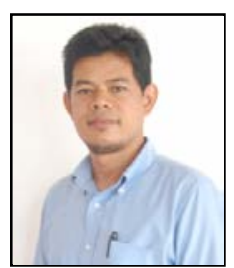

Waesomesudin Waedorkor was born in Pattan province, Thailand on July 24, 1979. He completed a bachelor of science degree in information technology and a master degree in management of information technology from Prince of Songkla University (PSU) HatYai, Thailand. Currently, He is a Ph.D student of the Department of Computer Engineering, Faculty of Engineering, PSU. His current research interests are in information engineering, management of information technology, dynamic adaptive streaming media, network management and mobile edge cloud computing.

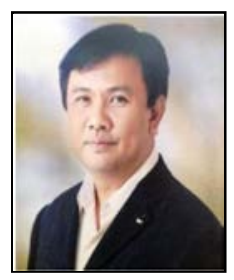

Suntorn Witosurapot is an assistant professor in the Department of Computer Engineering, Faculty of Engineering, in Prince of Songkla University (PSU), HatYai, Thailand. He received the bachelor and master degrees in electrical engineering from PSU, Thailand and Ph.D. degree from Swinburne University of Technology, Melbourne, Victoria, Australia, with the thesis topics related to resolving network resource competition in the Internet. His research interests include web engineering and applications, model-based software engineering, and management of information technology. Currently, most of his research work revolves around information engineering in smart home network environment and smart grid infrastructure. 